\title{
Artemisinin derivative artesunate induces radiosensitivity in cervical cancer cells in vitro and in vivo
}

Judong Luo ${ }^{1,2 \dagger}$, Wei Zhu ${ }^{1 \dagger}$, Yiting Tang ${ }^{1 \dagger}$, Han Cao ${ }^{1}$, Yuanyuan Zhou' ${ }^{1}$, Rong Ji ${ }^{1}$, Xifa Zhou' ${ }^{2}$, Zhongkai Lu Hongying Yang ${ }^{1}$, Shuyu Zhang ${ }^{1}$ and Jianping Cao ${ }^{1,3^{*}}$

\begin{abstract}
Objective: Cervical cancer is the third most common type of cancer in women worldwide and radiotherapy remains its predominant therapeutic treatment. Artesunate (ART), a derivative of artemisinin, has shown radiosensitization effect in previous studies. However, such effects of ART have not yet been revealed for cervical cancer cells.
\end{abstract}

Methods: The effect of ART on radiosensitivity of human cervical cancer cell lines HeLa and SiHa was assessed using the clonogenic assay. Cell cycle progression and apoptosis alterations were analyzed by flow cytometry. For in vivo study, HeLa or SiHa cells were inoculated into nude mice to establish tumors. Tissues from xenografts were obtained to detect the changes of microvessel density, apoptosis and cell cycle distribution. Microarray was used to analyze differentially expressed genes.

Results: ART increased the radiosensitivity of HeLa cells (SER $=1.43, P<0.001$ ) but not of SiHa cells. Apoptosis and the G2-M phase transition induced by X-ray irradiation (IR) were enhanced by ART via increased Cyclin B1 expression in HeLa cells. Tumor growth of xenografts from HeLa but not SiHa cells was significantly inhibited by irradiation combined with ART (tumor volume reduction of $72.34 \%$ in IR + ART group vs. $41.22 \%$ in IR group in HeLa cells and $48.79 \%$ in IR + ART group vs. $44.03 \%$ in IR alone group in SiHa cells). Compared with the irradiated group, cell apoptosis was increased and the G2/M cell cycle arrest was enhanced in the group receiving irradiation combined with ART. Furthermore, compared with radiation alone, X-ray irradiation plus ART affected the expression of 203 genes that function in multiple pathways including RNA transport, the spliceosome, RNA degradation and p53 signaling.

Conclusion: ART potently abrogates the G2 checkpoint control in HeLa cells. ART can induce radiosensitivity of HeLa cells in vitro and in vivo.

Keywords: Artesunate (ART), Cervical cancer, Radiosensitivity, Cell cycle, Apoptosis

\section{Introduction}

Cervical cancer used to be the number 1 killer of female human beings who suffered from cancer, with the development of general survey technique, the morbidity of cervical cancer decreased recently. However, cervical cancer remains the third most common type of cancer

\footnotetext{
* Correspondence: jpcao@suda.edu.cn

${ }^{\dagger}$ Equal contributors

'School of Radiation Medicine and Protection and Jiangsu Provincial Key Laboratory of Radiation Medicine and Protection, Soochow University, Suzhou 215123, China

${ }^{3}$ School of Radiation Medicine and Protection, Medical College of Soochow University, 199 Ren'ai Rd, Suzhou 215123, China

Full list of author information is available at the end of the article
}

in women worldwide, especially in the medical underdeveloped areas, and radiotherapy is a common treatment for this gynecological malignancy [1]. Radiotherapy for cancer of the cervix can be external or/and internal, which treats cervical cancer by using high-energy rays and destroy the cancer cells while doing as little harm as possible to normal cells. However, radioresistance remains one of the major reasons for clinical failure of radiotherapy [2]. Thus, to improve the efficacy of radiotherapy for cervical cancer, the combination of radiation with additional radiosensitizing agents is needed.

Artemisinin, a chemical compound derived from the sweet wormwood plant (Artemisia annua), has been well

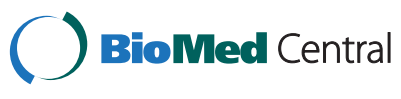

(c) 2014 Luo et al.; licensee BioMed Central Ltd. This is an Open Access article distributed under the terms of the Creative Commons Attribution License (http://creativecommons.org/licenses/by/2.0), which permits unrestricted use, distribution, and reproduction in any medium, provided the original work is properly credited. The Creative Commons Public Domain Dedication waiver (http://creativecommons.org/publicdomain/zero/1.0/) applies to the data made available in this article, unless otherwise stated. 
established to successfully treat malaria and viruses in humans for years. Various derivatives of artemisinin, including artesunate, artemether, dihydroartemisinin (DHA) and arteether have been identified [3,4]. Artesunate (ART) is a semi-synthetic derivative of artemisinin and a more effective antimalarial agent [5]. ART reveals remarkable activity against otherwise multidrug-resistant Plasmodium falciparum and P. vivax malaria. ART has also showed anticancer activity against a variety of cancer cells, especially in leukemia and colon cancer cell lines. The anticancer activity of ART is associated with multiple mechanisms, including reactive oxygen species (ROS), oxidative DNA damage, sustained DNA double-strand breaks and apoptosis [6-8].

Artemisinin and its derivative dihydroartemisinin have shown radiosensitizing effect in cervical cancer cells $[9,10]$. Recently, the ART has been implicated as an effective radiosensitiser in glioblastoma cells by decreasing survivin expression [11] and in lung cancer cells via increasing NO production [12]. However, whether such an effect of ART exists in cervical cancer cells remains elusive. This study aims to investigate the radiosensitizing effects of ART and underlying mechanisms in vitro and in vivo. Our results show that artesunate selective radiosensitization of human cervical cancer HeLa cells through abrogation of radiationinduced G2 block and cell apoptosis.

\section{Materials and methods \\ Reagents and cell culture}

ART was purchased from Sigma Chemical Co. (Sigma Chemical Co: St. Louis, MO) and was dissolved in dimethylsulfoxide (DMSO, Solon, $\mathrm{OH}$ ) to $10 \mathrm{mmol} / \mathrm{L}$ as stock solution $\left(-20^{\circ} \mathrm{C}\right.$ stocked), and diluted by DMEM to final concentration. For in vivo studies, ART was diluted with sterile PBS at $5 \mathrm{mg} / \mathrm{ml}$ before each administration.

The human cervical cancer cell lines HeLa and SiHa were kind gifts from Prof. Saijun Fan, Georgetown University. These cells were maintained in DMEM supplemented with $10 \%$ FBS and antibiotics (100 units/ml penicillin G, 100 units/ml streptomycin sulfate; Gibco, Grand Island, $\mathrm{NY}$ ). Cells were grown in a $37^{\circ} \mathrm{C}$ incubator with $5 \% \mathrm{CO}_{2}$.

\section{Cytotoxicity assay}

Cells $\left(2 \times 10^{3}\right)$ were seeded into 96-well plates in $100 \mu \mathrm{l}$ of DMEM medium and were incubated for $24 \mathrm{~h}$, and then the cells were treated with indicated concentrations of ART followed by incubated with $200 \mu \mathrm{g} / \mathrm{ml}$ MTT (3-(4,5-dimethylthiazol-2-yl)-2,5-diphenyl-2H-tetrazolium bromide, Sigma) for $4 \mathrm{~h}$. The reaction product was dissolved in DMSO. Absorbance was measured at background wavelength of $570 \mathrm{~nm}$, reference wavelength of $630 \mathrm{~nm}$ using a microplate reader. Three independent experiments were done in triplicate.

\section{Clonogenic assay}

Clonogenic assay was performed as described previously [10]. Cells were seeded into six-well plates at 500-2,000 cells/well depending on the dose of radiation. Twentyfour hours after seeding, cells were treated with ART or DMSO for $24 \mathrm{~h}$. Cells were exposed to various doses $(0,2$, 4, 6 and $8 \mathrm{Gy)}$ of X-rays irradiation from linear accelerators (Varian, USA) at a dose rate of $2 \mathrm{~Gy} / \mathrm{min}$; a $1.5-\mathrm{cm}$ bolus was used as a compensator. After radiation, drugcontaining media was immediately replaced by fresh DMEM. The cells were then grown from 7-12 days to allow for colony formation and subsequently fixed and stained using crystal violet. Colonies consisting of 50 or more cells were counted as clones.

\section{Measurement of apoptosis}

Cells were treated with ART for $24 \mathrm{~h}$ prior to treatment with 2 or 6 Gy irradiation. Apoptosis was measured using propidium iodide (PI)/Annexin-V double staining following manufacturer's instructions (Keygen Biotech, Nanjing, China). Cells were harvested $24 \mathrm{~h}$ after treatment with ART; apoptotic fractions were measured using flow cytometry (Beckman, USA). The Annexin-V+/PI- cells are early in the apoptotic process, the Annexin-V+/PI+ cells indicating late apoptosis. The percentage of both kinds of cells was counted. The Annexin-V-/PI + cells are considered to be necrotic cells.

For tissue samples, $5 \mu \mathrm{m}$ xenograft sections were deparaffinized in xylene and hydrated in decreasing concentrations of ethanol, and the terminal deoxynucleotidyl transferase dUTP nick-end labeling (TUNEL) assay was performed following manufacturer's instructions (Keygen Biotech, Nanjing, China). Ten random fields from 4 slides per group were examined. TUNEL-positive brown nuclei within tissues were counted. Data were expressed as the percentage of apoptotic cells per field.

\section{Cell cycle progression analysis}

Cells were treated with ART for $24 \mathrm{~h}$. Cells were then changed with fresh medium and irradiated the indicated doses. $24 \mathrm{~h}$ after irradiation, both floating and attached cells were harvested and analyzed using the procedures described previously (10). For flow cytometry, 10,000 cells per sample were collected (Beckman, USA).

For cell cycle analyses of tissue samples, tissue specimens were taken from nude mice and mixed with $200 \mu \mathrm{l}$ $0.25 \%$ trypsin and EDTA (1:1), stirred $1 \mathrm{~min}$ at room temperature and then filtered with a $70 \mu \mathrm{m}$ nylon net. Tumor cells were collected and pooled with the cells floating in the medium. Cell suspensions were centrifuged $5 \mathrm{~min}$ at $1,500 \mathrm{rpm}$, room temperature, then washed and fixed with ethanol at $4^{\circ} \mathrm{C}$ overnight. All samples were then washed with PBS and resuspended in PI $(50 \mu \mathrm{g} / \mathrm{mL})$ and RNase A $(20 \mu \mathrm{g} / \mathrm{mL})$ in PBS for $30 \mathrm{~min}$ 
at room temperature. Stained cells were analyzed by flow cytometry (Beckman, USA).

\section{Western blot}

Cells were treated for $24 \mathrm{~h}$ with ART. Cells were then changed with fresh medium and irradiated at the indicated doses. The cells were then washed twice with ice-cold PBS and directly lysed in $200 \mu \mathrm{l}$ of cell lysis buffer. Tumors from nude mice were resected, homogenized and lysed. Western blot was performed as described previously [13]. Primary antibodies against Wee1, Cyclin B1, P53 and Cdc2 were all obtained from Santa Cruz Biotechnology (Santa Cruz, CA) and used at a 1:1,000 -1:2,000 dilution. $\beta$-Actin was used as the loading control and detected using a mouse monoclonal anti- $\beta$-actin antibody (Santa Cruz Biotechnology).

\section{Xenograft studies of nude mice}

Four-week-old male outbred BALB/c mice were purchased from Shanghai SLAC Laboratory Animal Co., Ltd. (Shanghai, China), and kept under specific pathogen-free conditions. HeLa or $\mathrm{SiHa}$ cells $\left(2 \times 10^{6}\right)$ were suspended in $100 \mu \mathrm{l}$ PBS and then inoculated subcutaneously into each posterior flank region of $\mathrm{BALB} / \mathrm{c}$ nude mice. When the average volume of tumor achieved $5 \mathrm{~mm} \times 5 \mathrm{~mm} \times 5 \mathrm{~mm}$, mice were randomized into four groups as follows ( 5 animals per group): 1) Control group (no radiation, injection of $200 \mu \mathrm{l}$ sterile PBS once a day for 7 days), 2) ART alone group (no radiation, injection of ART once a day for 7 days at $100 \mathrm{mg} / \mathrm{kg} /$ day with a total volume of $200 \mu \mathrm{l}$ ), 3) Radiation (IR) alone group (injection of $200 \mu \mathrm{l}$ sterile PBS for 7 days and radiated at a dose of $10 \mathrm{~Gy}$ at the 7 th day one hour after the last PBS was injected, or 4) IR plus ART treatment group (injection of $200 \mu \mathrm{l}$ ART once a day for 7 days at $100 \mathrm{mg} / \mathrm{kg} /$ day and radiated at a dose of $10 \mathrm{~Gy}$ on the 7 th day after ART treatment). Both PBS and ART were intraperitoneal injected.

Tumor diameters were measured at regular intervals with digital calipers, and the tumor volume in $\mathrm{mm}^{3}$ was calculated using the formula: volume $=(\text { width })^{2} \times$ length $/$ 2. A tumor growth curve was constructed and data are presented as means \pm SEM. Animals were sacrificed 21 days after the first inoculation. All the animal study were approved by the Animal Experimentation Ethics Committee of Soochow University.

\section{Immunohistochemistry (IHC)}

Immunohistochemistry of CD 31 was performed as described previously [13]. Fetal lung tissue was used as a positive control, and omission of primary antibody was performed as a negative control.

\section{Microarray analysis of gene expression}

Total RNA was extracted from cells or tissues with Trizol reagent (Invitrogen, Carlsbad, CA). Microarraybased mRNA expression profiling was performed using the Roche-NimbleGen (135 K array) Array (Roche, WI). The microarrays contained approximately 45,033 assay probes corresponding to all of the annotated human mRNA sequences (NCBI HG18, Build 36). Total RNA labeling and hybridization were performed using standard conditions according to manufacturer instructions. Genes with an absolute fold change of 5 or greater were subsequently subjected to pathway analysis using Ingenuity Pathway Analysis (Redwood City, CA).

\section{Statistical analysis}

Data were expressed as the mean \pm standard error of the mean (SEM) of at least three independent experiments. Standard error bars are included for all data points. The data were then analyzed using Student's t-test when only two groups were present, or assessed by one-way analysis of variance (ANOVA) when more than two groups were compared. The interaction between ART and radiation was tested using two-way ANOVA. The sensitizer enhancement ratios (SER) were measured using Sigmaplot software according to the multi-target single hit model. Differences were considered statistically significant when $P<0.05$.

\section{Results}

\section{ART induces cytotoxicity in human cervical cancer cells}

To evaluate the anticancer effect of ART on cultured human cervical cancer cells, HeLa and SiHa cells were treated with different concentrations of ART. The OD value of cells treated without ART were regarded as control $(100 \%)$ and then the cell groups treated with different concentration of ART were versus with control. The MTT assay revealed that the inhibitory effects elicited by ART were dependent on its concentration (Figure 1A and B). The fifty percent inhibition concentration (IC50) of ART against HeLa and $\mathrm{SiHa}$ cells was $5.47 \mu \mathrm{mol} / \mathrm{L}$ and $6.34 \mu \mathrm{mol} / \mathrm{L}$, respectively. To evaluate the sensitizing ability of ART on cervical cancer cells to irradiation, moderately toxic doses that reduced cell viability to approximately $90 \%$ were used. ART induced approximately $10 \%$ inhibition of HeLa cell viability with the concentration of $2.0 \mu \mathrm{mol} / \mathrm{L}$, which was comparable to the effect of $2.5 \mu \mathrm{mol} / \mathrm{L}$ ART on SiHa cells. These concentrations were chosen for subsequent experiments.

\section{ART increases the radiosensitivity in HeLa but not SiHa cells}

To investigate the influence of ART on the radiosensitivity of HeLa and SiHa cells, we performed an in vitro clonogenic cell survival assay. HeLa cells pretreated with 

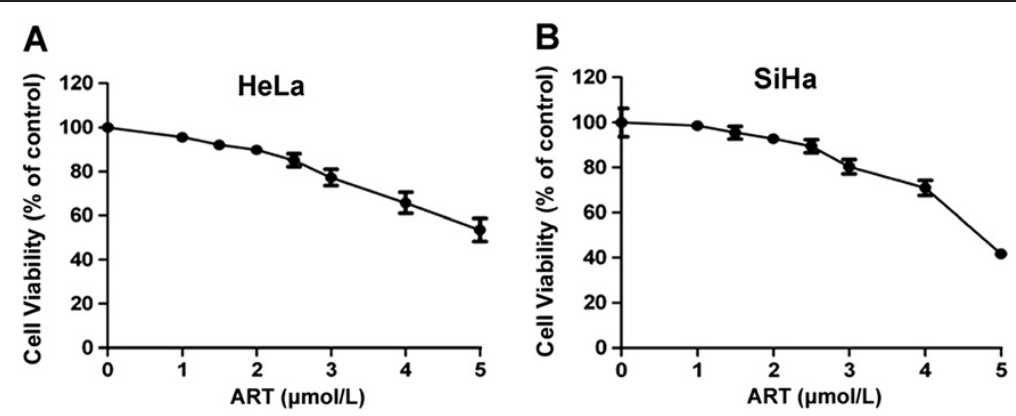

Figure 1 Cytotoxicity of ART on HeLa and SiHa cells. The ART-induced cytotoxicity in (A) HeLa and (B) SiHa cells. HeLa and SiHa cells were exposed to indicated concentrations of ART for $24 \mathrm{~h}$. Cell survival was assessed using an MTT assay. The data are shown as the mean values \pm the standard error of the mean (SEM) for three independent experiments.

$2.0 \mu \mathrm{mol} / \mathrm{L}$ ART plus X-ray irradiation exhibited significantly lower clonogenic survival fractions than cells treated with radiation alone. The sensitizer enhancement ratio (SER) was 1.43 for cells treated with radiation plus ART, compared to cells treated with radiation alone (Figure 2A). SiHa cells treated with radiation plus $2.5 \mu \mathrm{mol} / \mathrm{L}$ ART exhibited a SER of 1.03 (Figure 2B). The data were further analyzed using two-way ANOVA to test the interaction effect between ART and radiation. Our results indicated that interaction between ART and radiation was statistically significant $(P<0.001)$ for
HeLa cells, suggesting that ART treatment sensitized cells to X-ray irradiation. However, in $\mathrm{SiHa}$ cells, the interaction effect between ART and radiation was not statistically significant $(P>0.05)$. Taken together, these results demonstrated that treatment with ART could increase the radiosensitivity of human HeLa cells but not of SiHa cells.

We further investigated whether the difference between HeLa and SiHa cells in the ART-induced radiosensitizing effect is due to p53 status and expression level. HeLa cells with mutant p53 were transfected with a p53-overexpression vector (pcDNA3.1-p53). As shown

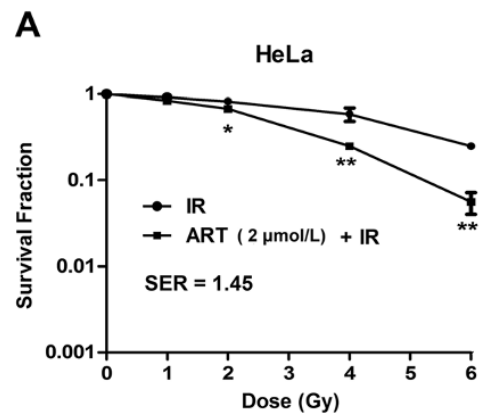

C

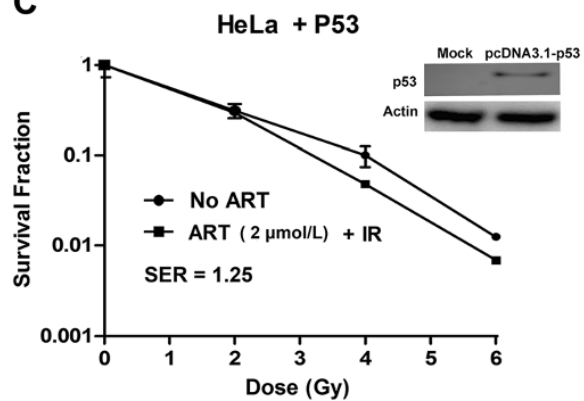

B

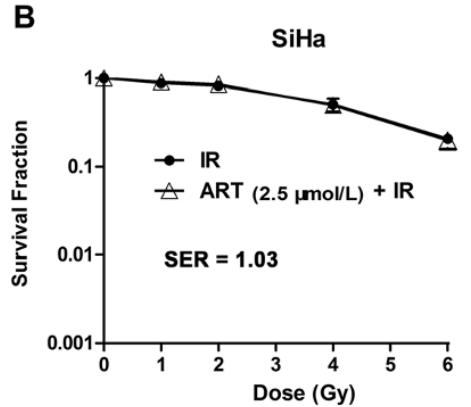

D

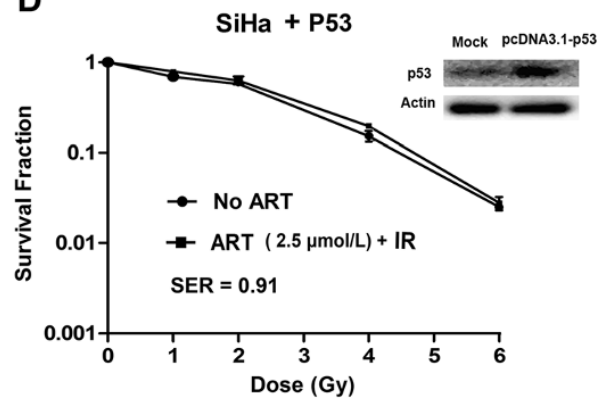

Figure 2 The ART-induced radiosensitivity in HeLa (A) and SiHa (B) cells. (C) and (D) The ART-induced radiosensitivity in wild-type p53-overexpression HeLa and SiHa cells. Western blot of p53 and internal control in pcDNA3.1- or pcDNA3.1-p53-transfected HeLa (C) and SiHa (D) cells. Clonogenic cell survival curves were generated for HeLa and SiHa cells that were treated with the indicated concentrations of ART for $24 \mathrm{~h}$ and then were exposed to 2, 4,6 or $8 \mathrm{~Gy}$ IR. The survival data were normalized to those of the unirradiated control group. The data are shown as mean \pm SEM for three independent experiments. The SER was calculated for HeLa or SiHa cells that were treated with 2.0 or $2.5 \mu$ mol/L ART prior to $X$-ray irradiation according to the multi-target single hit model. Values shown are the mean \pm SEM for three independent experiments. ${ }^{*} P<0.05 ;{ }^{*} P<0.01$, compared with irradiated cells. 
in Figures $2 \mathrm{C}$, introduction of wild-type p53 could partially reduce the radiosensitivity caused by ART (SER = 1.25) in HeLa cells, indicating that p53 status is likely to be one of the reasons for the selective radiosensitizing effect of ART. However, forced overexpression of p53 in $\mathrm{SiHa}$ cells significantly reduced the radiosensitizing effect of ART (SER $=0.91$, Figure 2D), indicating different roles of p53 in the two types of cell lines.

\section{ART induces apoptosis and necrosis in Hela cells}

We next investigated whether reduced clonogenic survival upon combined treatment with ART and X-ray irradiation was associated with increased apoptosis and necrosis. As shown in Figure 3A and B, 6Gy X-ray irradiation induced apoptosis (Annexin-V+/PI- plus Annexin-V+/PI + cells) and necrosis (Annexin-V-/PI + cells) in both HeLa and $\mathrm{SiHa}$ cell lines, and 2Gy X-ray irradiation only induced apoptosis but not necrosis in the two cell lines. This is mainly because necrosis arises when exposed to high dosage. ART treatment further enhanced the apoptosis response of HeLa cells to 2 or 6 Gy of irradiation (IR + ART $22.71 \%$ vs. IR $12.26 \%$ at 2 Gy, $P<0.05$; IR + ART 59.92\% vs. IR $40.08 \%$ at $6 \mathrm{~Gy}, P<0.05$, Figure $3 \mathrm{~A})$. However, there is no difference of the apoptosis response between radiation plus ART and radiation alone group in $\mathrm{SiHa}$ cells (IR + ART $14.35 \%$ vs. IR $12.276 \%$ at 2 Gy, $P>0.05$; IR + ART $33.67 \%$ vs. IR $32.25 \%$ at 6 Gy, $P>0.05$ ). The percentage of necrotic cells was similar between ART-treated and control cells in both cell lines (Figure 3A and B). Taken together, these results demonstrate that ART augments apoptotic cell death of HeLa cells in response to irradiation.

\section{ART combined with X-ray irradiation modulates cell cycle progression}

DNA damage by radiation activates checkpoint pathways that inhibit the progression of cells through G1 and G2 phases and delay progression through $\mathrm{S}$ phase. These checkpoints provide cells with enough time to repair damaged DNA prior to resuming cell cycle progression $[14,15]$. To determine whether the observed ART-induced radiosensitization was associated with changes in cell cycle progression, HeLa and SiHa cells were cultured in DMEM without serum for $24 \mathrm{~h}$ prior to the addition of ART, alone or combined with 2 or 6 Gy X-ray irradiation. As shown in Figures 4A and B, radiation induced a G2 arrest in the p53-mutant HeLa cells and a G1 arrest in p53 wild-type $\mathrm{SiHa}$ cells. Compared with irradiated cells, cells treatment with ART plus X-ray irradiation showed decreased the population of $\mathrm{G} 2 / \mathrm{M}$ arrest in $\mathrm{HeLa}$ (but not $\mathrm{SiHa}$ ) cells (a reduction of $20.77 \%$ at 2 Gy and $36.24 \%$ at 6 Gy, $P<$ 0.05). This result clearly indicates that ART abrogates the G2 checkpoint but elicits no effect on the G1 checkpoint.

As Wee1, Cdc2 and Cyclin B1 play critical roles in the control of the G2/M transition of the cell cycle progression [16-18]. We then investigated whether ART pretreatment modulated the expression of Wee1, Cyclin B1 or Cdc2 after X-ray irradiation of HeLa and $\mathrm{SiHa}$ cells. As shown in Figure 4C, 6 Gy X-ray irradiation induced the expression of Wee1. The addition of ART prior to irradiation resulted in decreased Wee1 and increased Cyclin B1 expression in HeLa cells (Figure 4C, left panel). In contrast, relative expression of $\mathrm{Cdc}$, Wee1 and Cyclin B1 remained unaltered in SiHa cells. These results indicated that combined treatment of ART and radiation reduced Wee1 and increased Cyclin B1 expression, abrogating the $G 2 / \mathrm{M}$ arrest induced by radiation in HeLa cells.

\section{Irradiation combined with ART inhibits the growth of HeLa xenografts}

As shown in Figure 5A and 6B, mice treated with ART alone yielded similar results as the control group. Compared with the control group, the tumor volume of mice treated with radiation alone was reduced by $41.22 \%$. Comparatively, HeLa xenografts that received combined treatment of 10 Gy radiation and ART exhibited much smaller tumors compared with mice that received radiation alone (volume reduction of $72.34 \%$ in IP + ART group), which suggests that ART can enhance radiosensitivity of HeLa xenografts. Consistent with in vitro studies, the radiosensitivity of $\mathrm{SiHa}$ xenografts was not significantly changed (volume reduction of $44.03 \%$ in IR alone group vs $48.79 \%$ in IR + ART group; $P>0.05$ ).

ART reduces microvessel density, promotes apoptosis and inhibits G2-M phase arrest in vivo

To explore the mechanism for the tumor growth difference, the expression of CD31 which is an endothelial cell surface molecule that can be used to visualize microvessel density was detected by IHC. The results showed that xenografts from the control group or from ART treatment alone shared similar expression levels of CD31 (Figure 5C and D). Xenografts from the irradiation plus ART treatment group displayed significantly less positive staining for CD31 in HeLa xenografts $(9.75 \%$ in IR + ART group vs. $24.25 \%$ in IR group, $P<0.05$, Figure 5C) but not in the SiHa counterparts (Figure 5D). These results indicated that irradiation combined with ART influences the formation of microvessels and consequent tumor growth in HeLa xenografts.

We then measured cell apoptosis using the TUNEL assay to further investigate the apoptotic status in xenografts,. As shown in Figures 6A and B, irradiation plus ART resulted in significantly higher apoptotic cell death in the xenografts compared with IR alone group (50.30\% in IR + ART group vs. $30.20 \%$ in IR group, $P<0.05$, 


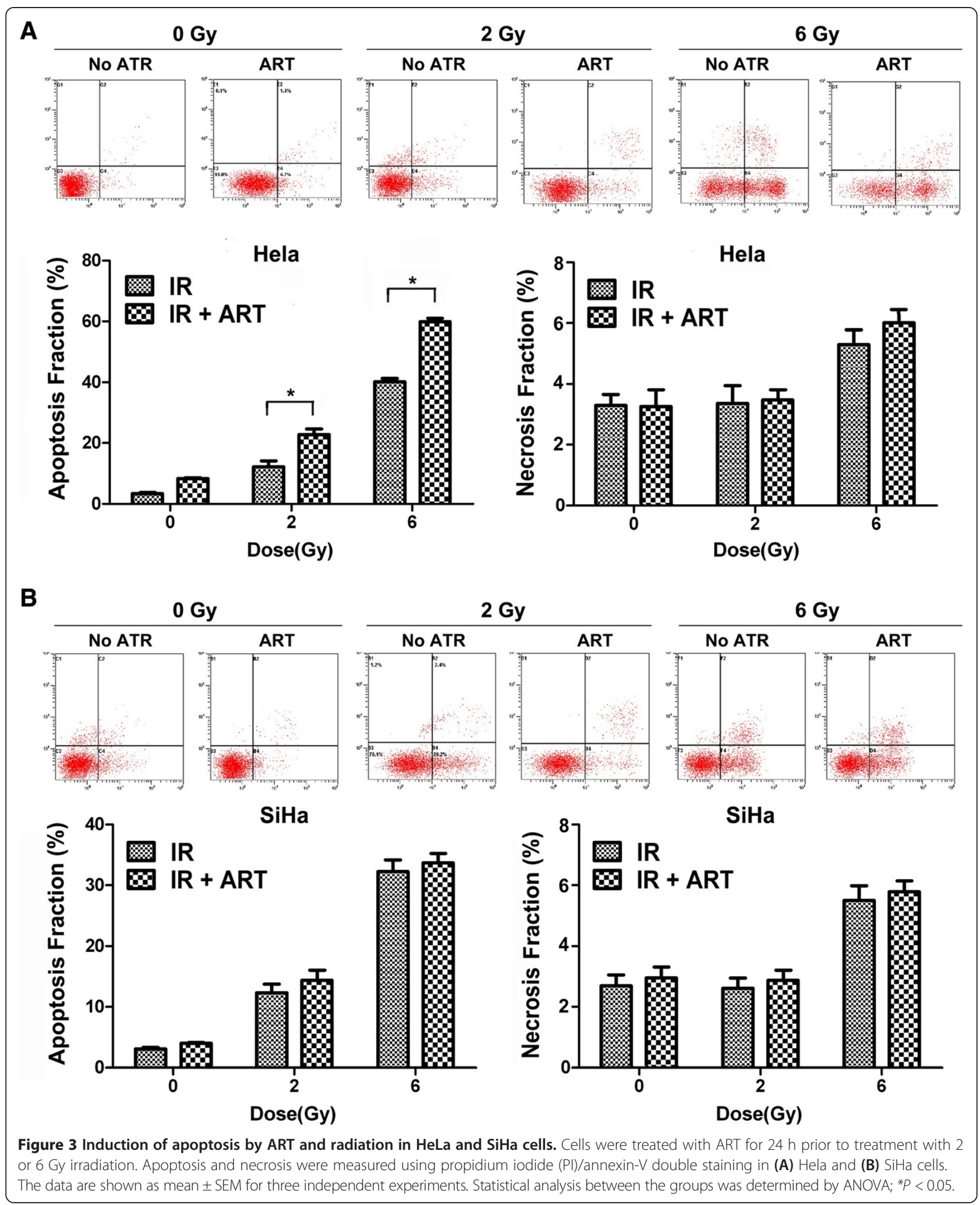

Figure 6B). These results indicated that irradiation combined with ART enhanced radiation-induced apoptosis in HeLa xenografts.
To determine whether irradiation plus ART influenced cell cycle progression in xenografts, flow cytometry was performed. The effect of combined radiation and ART 


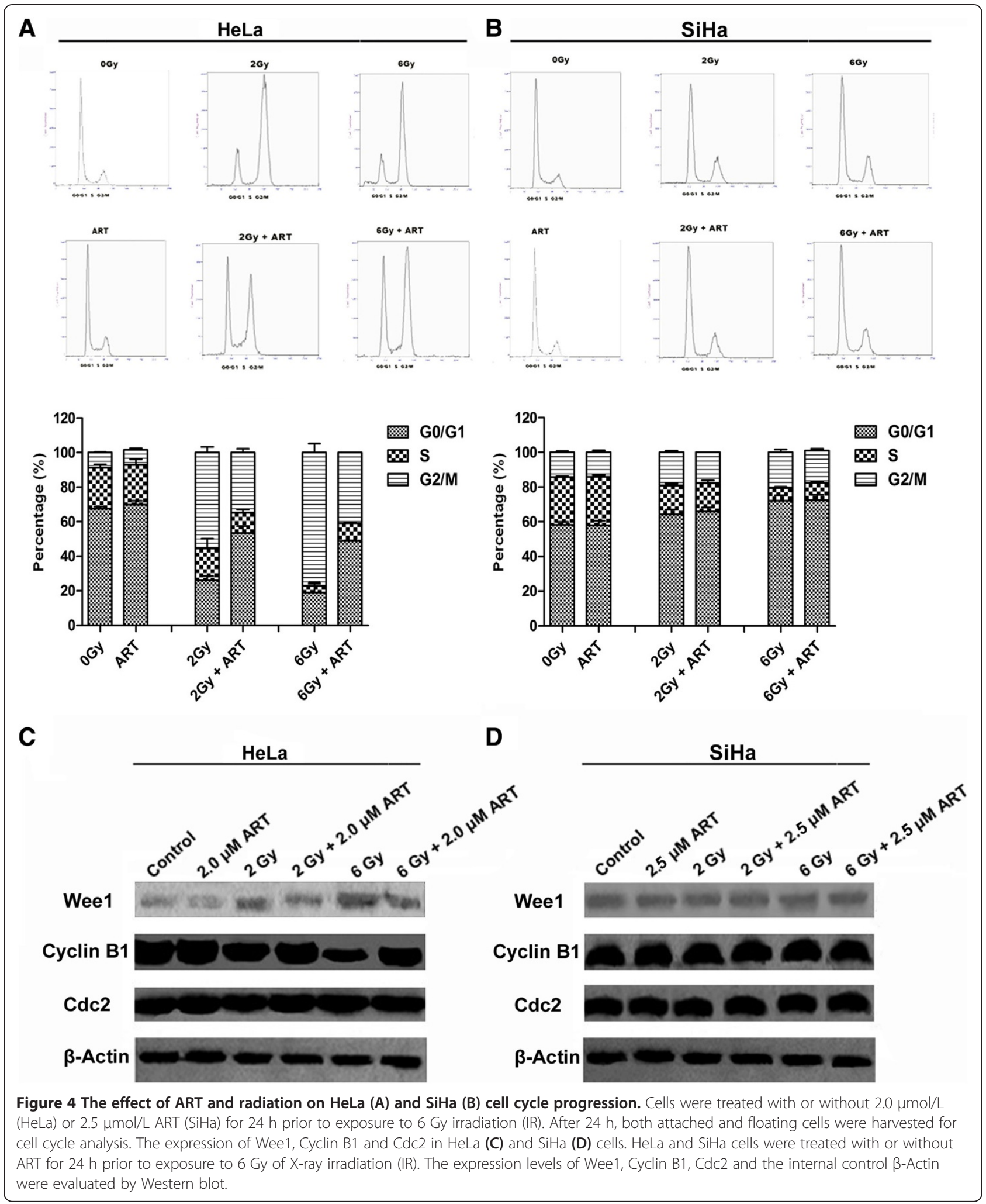

treatment on the cell cycle in tumors is shown in Figure 6C. Treatment with ART alone leads to no observed changes while comparing with the control group. However, compared with the radiation group, the percentage of tumor cells in G1 phase was significantly increased by 9.52\% $(P<0.05$, Figure $6 \mathrm{C})$ while the percentage of $\mathrm{G} 2$ 

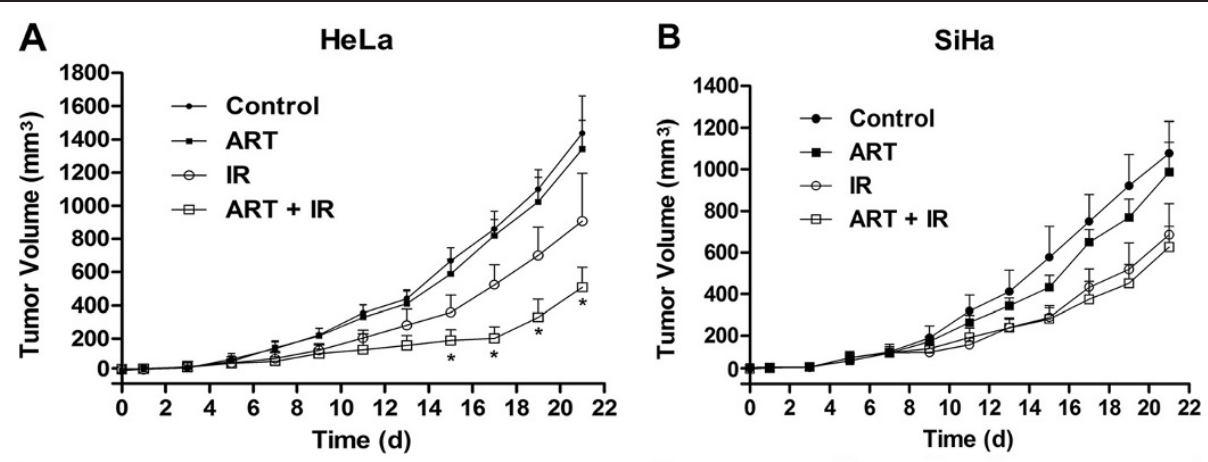

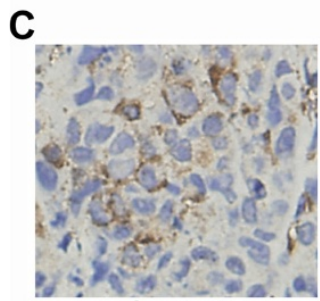

Control

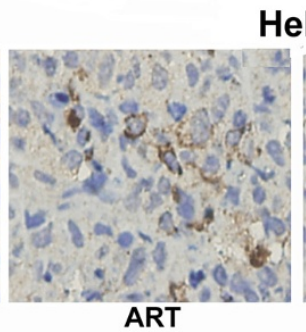

HeLa

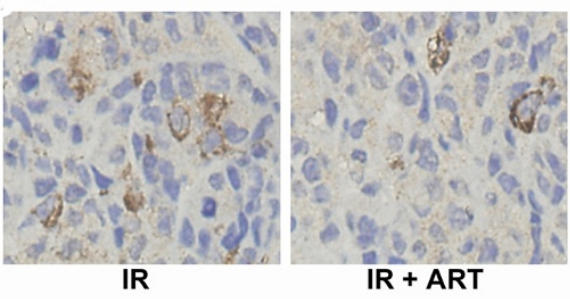

$\mathrm{SiHa}$

D

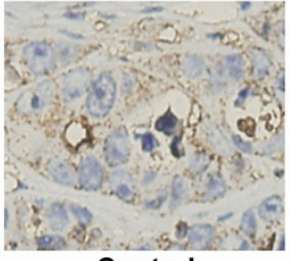

Control

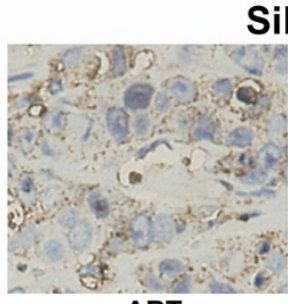

ART

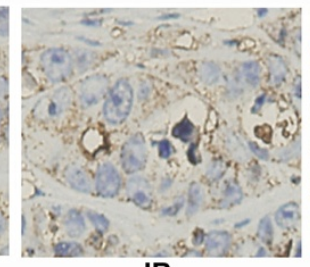

IR

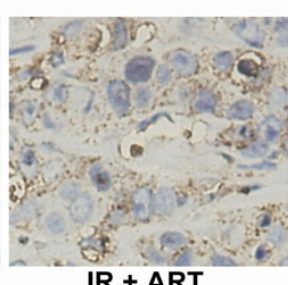

IR + ART

Figure $\mathbf{5}$ The effect of ART on the growth and CD31 expression of xenografts. Each group of mice was composed of five male nude mice. HeLa (A) or SiHa cells (B) were inoculated under the skin of nude mice. Tumor size was measured at 2 day intervals. ${ }^{*} P<0.05$, compared with $I R$ alone group. Data are presented as means \pm SEM. Representative IHC showing CD31-stained microvessels in the xenografts from HeLa (C) and SiHa cells (D). Scale bar represents $50 \mu \mathrm{m}$ (magnification, $\times 400$ ).

phase was significantly decreased by $13.24 \%(P<0.05$, Figure $6 \mathrm{C})$ in the radiation combined with ART group. It is likely that ART decreases G2 phase arrest of tumor cells after irradiation. We next examined the expression of Cyclin B1 and Cdc2 in the HeLa xenografts. As shown in Figure 6D, increased Cyclin B1 protein levels from xenografts were consistent with those in the previous in vitro study. However, the expression of Cdc2 was not changed in these xenografts, suggesting that the cell cycle changes may be triggered by Cyclin B1.

\section{ART increases radiosensitivity of HeLa cells via complex mechanisms}

To further analyze the underlying mechanisms responsible for ART-mediated radiosensitivity, we profiled gene expression between HeLa cells after either 6 Gy X-ray irradiation or the combination of $2.0 \mu \mathrm{mol} / \mathrm{L}$ ART prior to 6 Gy X-ray irradiation. Twenty-four hours after the treatments, a total of 203 genes (91 upregulated and 112 downregulated genes) were identified with differential expression of 5 -fold or more between the two groups of cells (Figure 7A and Table 1). Consistent with previous findings $[19,20]$, the administration of ART remarkably inhibited the expression of genes associated with hemoglobin and immunoglobulin. As expected, ART seems to modulate the radiosensitivity of HeLa cells via complex mechanisms. Pathway analysis revealed that ART treatment affected multiple pathways including RNA transport, the spliceosome, RNA degradation, p53 signaling and MAPK (Figure 7B and C).

\section{Discussion}

The anti-malarial ART has been reported to act against a variety of cancer cells including leukemia, lung and colon cancer cell lines [21-23]. Previously, we have reported that artemisinin and its analog dihydroartemisinin are effective radiosensitisers for cervical cancer cells $[9,10]$. In this study, we found that another artemisinin derivative, ART, increased the radiosensitivity and 


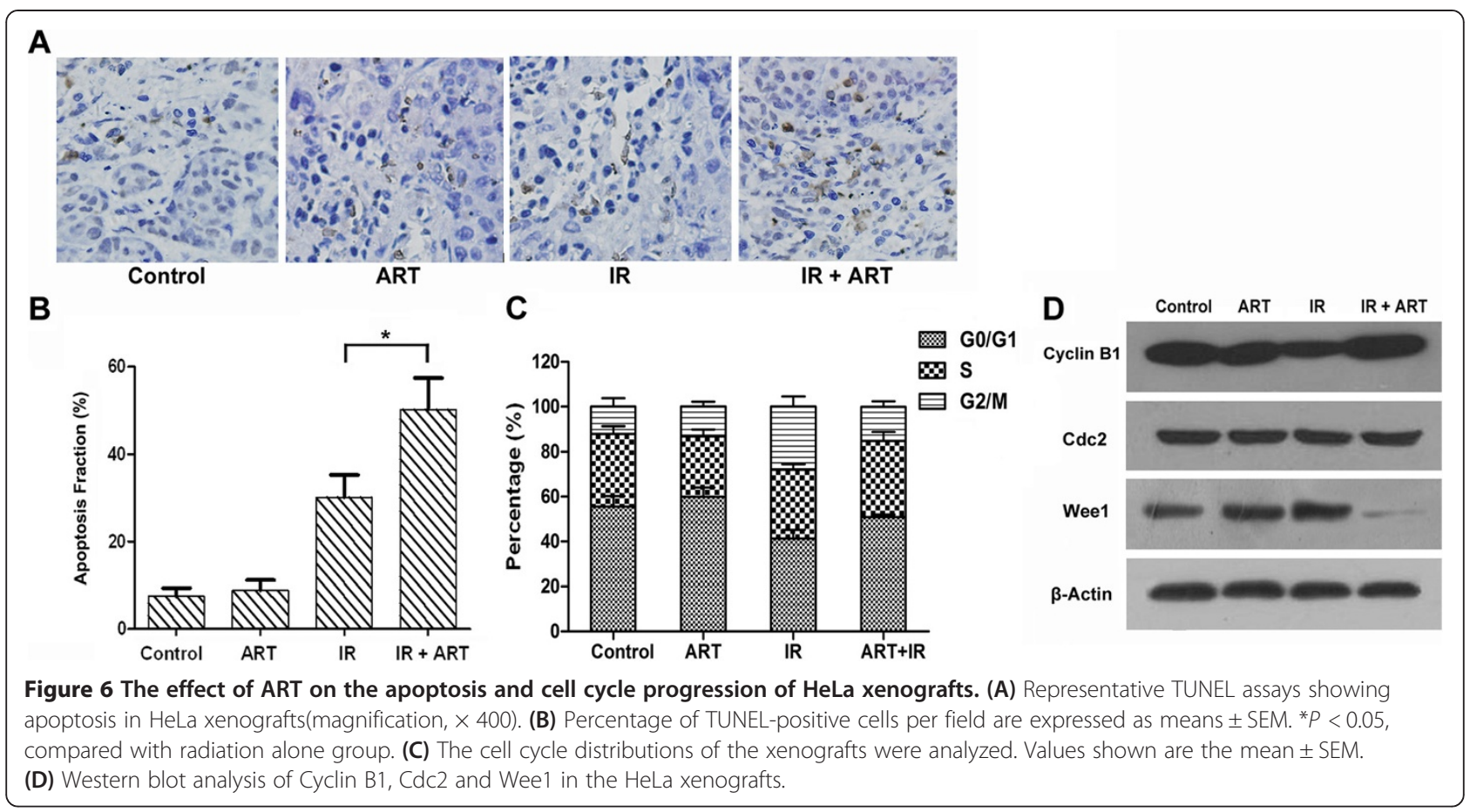

\section{A}

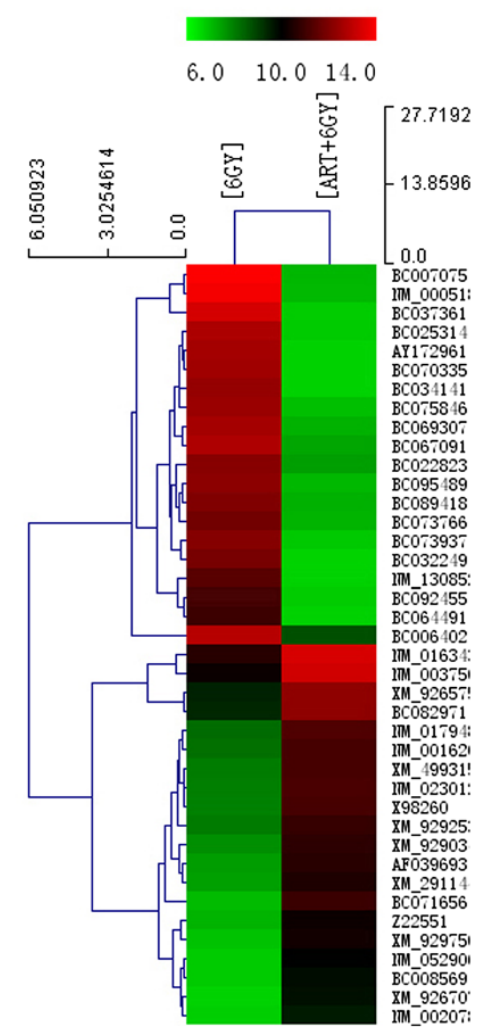

B

\section{Significant pathway of upregulated genes}

Ribosome biogenesis in eukaryotes - Homo sapiens (human) RNA transport - Homo sapiens (human) Spliceosome - Homo sapiens (human) Lysine degradation - Homo sapiens (human) Mismatch repair - Homo sapiens (human) RNA degradation - Homo sapiens (human) One carbon pool by folate - Homo sapiens (human) Aminoacyl-tRNA biosynthesis - Homo sapiens (human)

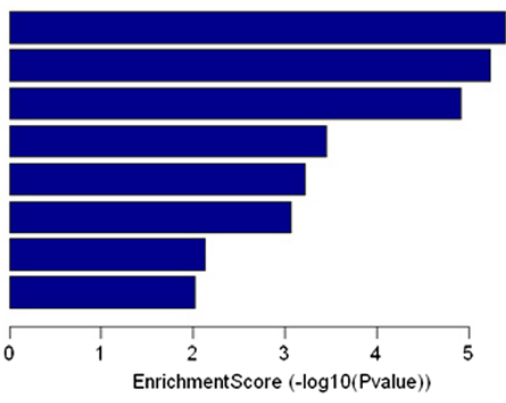

C

Significant pathway of downregulated genes

p53 signaling pathway - Homo sapiens (human) MAPK signaling pathway - Homo sapiens (human) Prostate cancer - Homo sapiens (human)

Pathways in cancer - Homo sapiens (human) Oxidative phosphorylation - Homo sapiens (human) Transcriptional misregulation in cancer - Homo sapiens (human) Adipocytokine signaling pathway - Homo sapiens (human) Colorectal cancer - Homo sapiens (human)

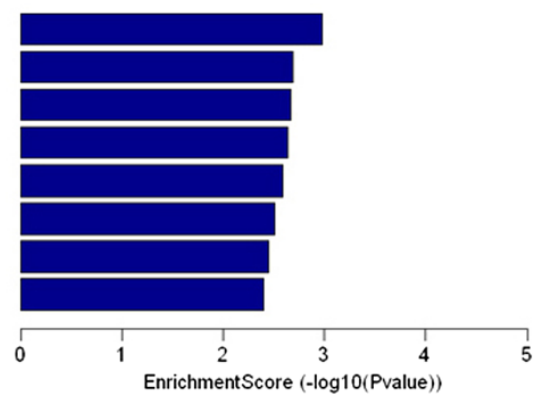

Figure 7 Predicted significant pathways involved. (A) Heat map of gene expression between HeLa cells after 6 Gy X-ray irradiation or $2.0 \mu \mathrm{mol} / \mathrm{L}$ ART prior to 6 Gy X-ray irradiation. (B) Predicted significant pathways for upregulated genes. (C) Predicted significant pathways for downregulated genes. 
Table 1 Microarray gene expression changes relative to irradiated HeLa cells (X-ray irradiation plus ART vs. X-ray irradiation alone)

\begin{tabular}{|c|c|c|c|}
\hline Gene name & Fold change upregulated & Chromosome & Description \\
\hline$\overline{\text { IMAA }}$ & 14.44 & chr16 & SLC7A5 pseudogene \\
\hline HSUP1 & 10.05 & chr20 & Similar to RPE-spondin \\
\hline FLJ11021 & 9.10 & chr12 & Similar to splicing factor, arginine/serine-rich 4 \\
\hline KIAA1904 & 8.98 & $\operatorname{chr} 22$ & KIAA1904 protein \\
\hline ZRF1 & 8.95 & chr7 & Zuotin related factor 1 \\
\hline SDCCAG10 & 8.61 & chr5 & Serologically defined colon cancer antigen 10 \\
\hline LOC442573 & 8.47 & chr7 & Similar to postmeiotic segregation increased 2-like 2 \\
\hline EIF3S10 & 8.42 & chr10 & Eukaryotic translation initiation factor 3 , subunit 10 theta \\
\hline KTN1 & 8.34 & chr14 & Kinectin 1 (kinesin receptor) \\
\hline LOC642617 & 8.23 & chr2 & Hypothetical protein LOC642617 \\
\hline LOC340089 & 7.98 & chr5 & Similar to nuclear pore membrane protein 121 \\
\hline GPR18 & 7.97 & chr13 & G protein-coupled receptor 18 \\
\hline LOC646074 & 7.93 & chr22 & Similar to nuclear pore membrane protein 121 \\
\hline NOL8 & 7.71 & chr9 & Nucleolar protein 8 \\
\hline AHNAK & 7.26 & chr11 & AHNAK nucleoprotein (desmoyokin) \\
\hline N/A & 7.16 & chr3 & Homo sapiens cDNA clone MGC:99509 IMAGE:3939369 \\
\hline GOLGA4 & 7.10 & chr3 & Golgi autoantigen, golgin subfamily a, 4 \\
\hline LOC646316 & 6.95 & chr4 & $\begin{array}{c}\text { Similar to Telomeric repeat binding factor } 1 \\
\text { interacting protein 2) }\end{array}$ \\
\hline CENPF & 6.92 & chr1 & Centromere protein F, 350/400 ka (mitosin) \\
\hline LOC643211 & 6.91 & chr2 & Hypothetical protein LOC643211 \\
\hline Gene name & Fold change downregulated & Chromosome & Description \\
\hline $\mathrm{HBB}$ & 116.84356 & chr11 & Hemoglobin, beta \\
\hline HBB & 110.22069 & chr11 & Hemoglobin, beta \\
\hline IGHG1 & 93.40286 & chr14 & Immunoglobulin heavy constant gamma 1 (G1m marker) \\
\hline N/A & 60.31211 & chr2 & Anti-rabies SOJA immunoglobulin kappa light chain mRNA \\
\hline IGHG1 & 59.625664 & chr14 & Immunoglobulin heavy constant gamma 1 (G1m marker) \\
\hline IGKC & 56.961563 & chr2 & Immunoglobulin kappa constant \\
\hline IGKC & 50.49672 & chr2 & Immunoglobulin kappa constant \\
\hline $\mathrm{HBD}$ & 43.09641 & chr11 & Hemoglobin, delta \\
\hline IGHG1 & 42.666557 & chr14 & Immunoglobulin heavy constant gamma 1 (G1m marker) \\
\hline IGHG1 & 40.846592 & chr14 & Immunoglobulin heavy constant gamma 1 (G1m marker) \\
\hline IGKC & 37.99056 & chr2 & Immunoglobulin kappa constant \\
\hline IGHA1 & 36.901497 & chr14 & Immunoglobulin heavy constant alpha 1 \\
\hline IGKC & 34.52912 & chr2 & Immunoglobulin kappa constant \\
\hline IGLV3-25 & 28.34214 & chr22 & Immunoglobulin lambda variable 3-25 \\
\hline PLUNC & 25.71026 & chr20 & Palate, lung and nasal epithelium carcinoma associated \\
\hline IGLV3-25 & 25.19829 & chr22 & Immunoglobulin lambda variable 3-25 \\
\hline LOC652848 & 24.885628 & chr14 & Similar to Ig heavy chain $\mathrm{V}$-II region $\mathrm{ARH}-77$ precursor \\
\hline N/A & 19.36516 & chr2 & Homo sapiens cDNA clone MGC:104455 IMAGE:30352955 \\
\hline N/A & 19.346416 & chr22 & Homo sapiens cDNA clone MGC:71261 IMAGE:4576612 \\
\hline $\mid \mathrm{GHG} 1$ & 17.280687 & chr14 & Immunoglobulin heavy constant gamma 1 (G1m marker) \\
\hline
\end{tabular}


promoted the apoptosis of p53-mutant HeLa cells but not of the wild-type p53 SiHa cells, both in vitro and in vivo. In HeLa cells, combined ART and radiation treatment decreased Wee1 but increased Cyclin B1 expression levels, impairing the irradiation-induced $\mathrm{G}_{2} / \mathrm{M}$ arrest. ART decreased the G2/M arrest induced by radiation, which most likely resulted in more irradiationdamaged cells entering mitosis. Moreover, the combined treatment of ART and irradiation increased apoptosis in cultured HeLa cells and HeLa xenografts. In the in vivo study, ART was given at a concentration of $100 \mathrm{mg} / \mathrm{kg} /$ day regularly for 7 days to achieve effective blood concentration in the nude mouse. Further investigation within other levels of ART should be tested.

ART has been reported to induce DNA damage response and repair genes, oncogenes, tumor suppressor genes and apoptosis-regulating genes [7]. DNA-damaging agents often induce cell cycle arrest in G1 or G2/M phases $[24,25]$, which are facilitated by checkpoint mechanisms that provide time for the repair of sub-lethal DNA damage prior to the resumption of cell cycle progression [26]. Therefore, the abrogation of the G2 checkpoint, which promotes premature mitotic entry and subsequent cell death, has emerged as a potential therapeutic strategy $[17,27]$. Cells in G2/M phase are particularly susceptible to the effects of radiation. Because of this, agents that alter cell cycle progression are often potent radiation modifiers [28]. In eukaryotic cells, Wee1 phosphorylates Cdc2 on Tyr-15 and inhibits its kinase activity, thereby preventing entry into mitosis [29-32]. Therefore, suppression of Wee1 can reduce Cdc2 $\mathrm{Tyr}-15$ phosphorylation and lead to the activation of $\mathrm{Cdc} 2$ kinase. Irradiation exposure can induce a G2/M arrest in HeLa cells through increased and decreased expression of Wee1 and Cyclin B1, respectively. We found that this cell cycle arrest and protein expression change was reversed by treatment with ART. The expression of Cdc2 exhibited no significant changes in either HeLa or $\mathrm{SiHa}$ cells that were treated with a combination of ART and irradiation, suggesting that ART might affect Cdc2 indirectly through Wee1. Wee1 is part of an intricate network of kinases and phosphatases that regulate the G2 checkpoint [33]; the abrogation of this checkpoint by Wee1 inhibition results in mitotic catastrophe. ART treatment resulted in similar abrogation of the radiation-induced G2 arrest in HeLa cells. However, ART elicited no significant inhibition effect on G1/S arrest, similarly to artemisinin $[9,10]$.

In this study, we found that ART exhibits different radiosensitizing effects between $\mathrm{HeLa}$ and $\mathrm{SiHa}$ cells. ART is reported to act via p53-dependent and -independent pathways in cancer cells [22]. The tumor suppressor and transcription factor p53 is a major regulator of cellular defense against neoplastic transformation and cancer development [34-37]. Defects in p53-dependent pathways are correlated with tumor resistance to radiation and chemotherapy [35]. The present study used paired p53-positive and p53-negative cancer cells to confirm the hypothesis that abrogation of the G2 checkpoint by targeting Cyclin B1 and Wee1 kinases represents an effective therapeutic approach against p53-null cancer cells. Wild-type p53 might protect the genome from accumulating DNA damage and transmitting genetic mutations to subsequent daughter cells [34-36]. However, forced expression of wildtype p53 could partially change the radiosensitizing effect of ART in HeLa cells, which suggests that p53 status is only one of the reasons for the selective radiosensitization of ART. Such a phenomenon is also observed for artemisinin and dihydroartemisinin $[9,10]$.

As expected, ART seems to modulate the radiosensitivity of HeLa cells via complex mechanisms by affecting multiple pathways including RNA transport, the spliceosome and RNA degradation. ART treatment also increased the transcripts of EIF3 (eukaryotic translation initiation factor 3), which induces apoptosis in multiple cancer cells [38]. However, we did not detect expression changes of Wee1 or Cyclin B1 in cells pretreated with ART, indicating that their expression changes may occur at the protein level.

In summary, ART enhances radiation-induced apoptosis and relieves the G2/M arrest in vitro and in vivo, which shows its promise as an effective radiosensitiser in cancer therapy. Clinical application of this anti-malarial drug can be expanded to complement radiotherapy for cancers.

\section{Abbreviations}

ART: Artesunate; DHA: Dihydroartemisinin; SER: Sensitizer enhancement ratios; CENPF: Centromere protein F; EIF3: Eukaryotic translation initiation factor 3.

\section{Competing interests}

The authors declare that they have no competing interests.

\section{Authors' contributions}

$J \mathrm{~L}$ and WZ carried out the molecular biology studies and drafted the manuscript. YT performed part of the molecular biology and drafted the figures. $H C, R J$ and $Y Z$ performed the animal experiment. SZ and $H Y$ performed the statistical analysis. JC, XZ and ZL participated in study design and modified the manuscript. All authors read and approved the final manuscript.

\section{Acknowledgements}

This work is supported by the National Natural Science Foundation of China (8117259714 and 81102078), Changzhou Social Development Project (CE20125026), the Key Programs of Natural Science Foundation of Jiangsu Educational Committee (11KJA310001) and the Priority Academic Program Development of Jiangsu Higher Education Institutions (PAPD).

\section{Author details}

${ }^{1}$ School of Radiation Medicine and Protection and Jiangsu Provincial Key Laboratory of Radiation Medicine and Protection, Soochow University, Suzhou 215123, China. ${ }^{2}$ Deaprtment of Radiotherapy, Changzhou Tumor Hospital, Soochow University, Changzhou 213001, China. ${ }^{3}$ School of Radiation Medicine and Protection, Medical College of Soochow University, 199 Ren'ai Rd, Suzhou 215123, China. 
Received: 9 October 2013 Accepted: 18 March 2014

Published: 25 March 2014

\section{References}

1. Siegel R, Ward E, Brawley O, Jemal A: Cancer statistics, 2011: the impact of eliminating socioeconomic and racial disparities on premature cancer deaths. CA Cancer J Clin 2011, 61:212-236.

2. Kim TJ, Lee JW, Song SY, Choi JJ, Choi CH, Kim BG, Lee JH, Bae DS: Increased expression of PAKT is associated with radiation resistance in cervical cancer. Br J Cancer 2006, 94:1678-1682.

3. Wang J, Huang L, Li J, Fan Q, Long Y, Li Y, Zhou B: Artemisinin directly targets malarial mitochondria through its specific mitochondrial activation. PLoS One 2010, 5:e9582.

4. Ase I, Lai H, Singh NP, Sasaki T: Anticancer properties of artemisinin derivatives and their targeted delivery by transferrin conjugation. Int J Pharm 2008, 354:28-33.

5. Efferth T, Romero MR, Wolf DG, Stamminger T, Marin JJ, Marschall M: The antiviral activities of artemisinin and artesunate. Clin Infect Dis 2008, 47:804-811.

6. Zhou C, Pan W, Wang XP, Chen TS: Artesunate induces apoptosis via a Bak-mediated caspase-independent intrinsic pathway in human lung adenocarcinoma cells. J Cell Physiol 2012, 227:3778-3786.

7. Berdelle N, Nikolova T, Quiros S, Efferth T, Kaina B: Artesunate induces oxidative DNA damage, sustained DNA double-strand breaks, and the ATM/ATR damage response in cancer cells. Mol Cancer Ther 2011, 10:2224-2233.

8. Jiang Z, Chai J, Chuang HH, Li S, Wang T, Cheng Y, Chen W, Zhou D: Artesunate induces G0/G1 cell cycle arrest and iron-mediated mitochondrial apoptosis in A431 human epidermoid carcinoma cells. Anticancer Drugs 2012, 23:606-613.

9. Gong XM, Zhang Q, Torossian A, Cao JP, Fu S: Selective radiosensitization of human cervical cancer cells and normal cells by artemisinin through the abrogation of radiation-induced G2 block. Int J Gynecol Cancer 2012, 22:718-724

10. Luo J, Chen X, Chen G, Zhou X, Lu X, Ling Y, Zhang S, Zhu W, Cao J: Dihydroartemisinin induces radiosensitivity in cervical cancer cells by modulating cell cycle progression. Saudi Med J 2009, 34:254-260

11. Reichert S, Reinboldt V, Hehlgans S, Efferth T, Rodel C, Rodel F: A radiosensitizing effect of artesunate in glioblastoma cells is associated with a diminished expression of the inhibitor of apoptosis protein survivin. Radiother Oncol 2012, 103:394-401.

12. Zhao Y, Jiang W, Li B, Yao Q, Dong J, Cen Y, Pan X, Li J, Zheng J, Pang X: Artesunate enhances radiosensitivity of human non-small cell lung cancer A549 cells via increasing NO production to induce cell cycle arrest at G2/M phase. Int Immunopharmacol 2011, 11:2039-2046.

13. Liu P, Zhou J, Zhu H, Xie L, Wang F, Liu B, Shen W, Ye W, Xiang B, Zhu X VEGF-C promotes the development of esophageal cancer via regulating CNTN-1 expression. Cytokine 2011, 55:8-17.

14. Fernet M, Megnin-Chanet F, Hall J, Favaudon V: Control of the G2/M checkpoints after exposure to low doses of ionising radiation: implications for hyper-radiosensitivity. DNA Repair (Amst) 2010, 9:48-57.

15. Niida $\mathrm{H}$, Nakanishi M: DNA damage checkpoints in mammals. Mutagenesis 2006, 21:3-9.

16. Mir SE, de Witt Hamer PC, Krawczyk PM, Balaj L, Claes A, Niers JM: In silico analysis of kinase expression identifies WEE1 as a gatekeeper against mitotic catastrophe in glioblastoma. Cancer Cell 2010, 18:244-257.

17. Perry JA, Kornbluth S: Cdc25 and Wee1: analogous opposites? Cell Div 2007, 2:12.

18. Dixon BP, Henry J, Siroky BJ, Chu A, Groen PA, Bissler JJ: Cell cycle control and DNA damage response of conditionally immortalized urothelial cells. PLoS One 2011, 6:e16595.

19. Pandey AV, Tekwani BL, Singh RL, Chauhan VS: Artemisinin, an endoperoxide antimalarial, disrupts the hemoglobin catabolism and heme detoxification systems in malarial parasite. J Biol Chem 1999, 274:19383-19388.

20. Messori L, Gabbiani C, Casini A, Siragusa M, Vincieri FF, Bilia AR: The reaction of artemisinins with hemoglobin: a unified picture. Bioorg Med Chem 2006, 14:2972-2977.

21. Efferth T, Dunstan H, Sauerbrey A, Miyachi H, Chitambar CR: The anti-malarial artesunate is also active against cancer. Int J Oncol 2001, 18:767-773.
22. Efferth T, Sauerbrey A, Olbrich A, Gebhart E, Rauch P, Weber HO, Hengstler JG, Halatsch ME, Volm M, Tew KD: Molecular modes of action of artesunate in tumor cell lines. Mol Pharmacol 2003, 64:382-394.

23. Hamacher-Brady A, Stein HA, Turschner S, Toegel I, Mora R, Jennewein N, Efferth T, Eils R, Brady NR: Artesunate activates mitochondrial apoptosis in breast cancer cells via iron-catalyzed lysosomal reactive oxygen species production. J Biol Chem 2011, 286:6587-6601.

24. Concin N, Stimpfl M, Zeillinger C, Wolff U, Hefler L, Sedlak J, Leodolter S, Zeillinger R: Role of $\mathrm{p} 53$ in G2/M cell cycle arrest and apoptosis in response to gamma-irradiation in ovarian carcinoma cell lines. Int J Oncol 2003, 22:51-57.

25. Branzei D, Foiani M: Regulation of DNA repair throughout the cell cycle. Nat Rev Mol Cell Biol 2008, 9:297-308.

26. Kawabe T: G2 checkpoint abrogators as anticancer drugs. Mol Cancer Ther 2004, 3:513-519.

27. Leijen $\mathrm{S}$, Beijnen JH, Schellens JH: Abrogation of the $\mathrm{G} 2$ checkpoint by inhibition of Wee-1 kinase results in sensitization of p53-deficient tumor cells to DNA-damaging agents. Curr Clin Pharmacol 2010, 5:186-191.

28. Liebmann J, Cook JA, Fisher J, Teague D, Mitchell JB: In vitro studies of Taxol as a radiation sensitizer in human tumor cells. J Natl Cancer Inst 1994, 86:441-446.

29. PosthumaDeBoer J, Wurdinger T, Graat HC, van Beusechem WW, Helder MN, van Royen BJ, Kaspers GJ: WEE1 inhibition sensitizes osteosarcoma to radiotherapy. BMC Cancer 2011, 11:156

30. Owens L, Simanski S, Squire C, Smith A, Cartzendafner J, Cavett V: Activation domain-dependent degradation of somatic Wee1 kinase. J Biol Chem 2010, 285:6761-6769.

31. Polidarova L, Sotak M, Sladek M, Pacha J, Sumova A: Temporal gradient in the clock gene and cell-cycle checkpoint kinase Wee1 expression along the gut. Chronobiol Int 2009, 26:607-620.

32. Gravett AM, Liu WM, Krishna S, Chan WC, Haynes RK, Wilson NL, Dalgleish AG: In vitro study of the anti-cancer effects of artemisone alone or in combination with other chemotherapeutic agents. Cancer Chemother Pharmacol 2011, 67:569-577.

33. Raleigh JM, O'Connell MJ: The G(2) DNA damage checkpoint targets both Wee1 and Cdc25. J Cell Sci 2000, 113(Pt 10):1727-1736.

34. Efeyan A, Serrano M: p53: guardian of the genome and policeman of the oncogenes. Cell Cycle 2007, 6:1006-1010.

35. Kastan MB, Berkovich E: p53: a two-faced cancer gene. Nat Cell Biol 2007, 9:489-491

36. Eriksson D, Stigbrand T: Radiation-induced cell death mechanisms. Tumour Biol 2010, 31:363-372.

37. Lu C, El-Deiry WS: Targeting p53 for enhanced radio- and chemo-sensitivity. Apoptosis 2009, 14:597-606.

38. Shi J, Hershey JW, Nelson MA: Phosphorylation of the eukaryotic initiation factor $3 \mathrm{f}$ by cyclin-dependent kinase 11 during apoptosis. FEBS Lett 2009, 583:971-977.

doi:10.1186/1748-717X-9-84

Cite this article as: Luo et al:: Artemisinin derivative artesunate induces radiosensitivity in cervical cancer cells in vitro and in vivo. Radiation Oncology 2014 9:84

\section{Submit your next manuscript to BioMed Central and take full advantage of:}

- Convenient online submission

- Thorough peer review

- No space constraints or color figure charges

- Immediate publication on acceptance

- Inclusion in PubMed, CAS, Scopus and Google Scholar

- Research which is freely available for redistribution 